\title{
Design of Reconfigurable Stacked Patch Microstrip Antenna (Rspma) at 1.8 Ghz and $2.3 \mathrm{Ghz}$
}

${ }^{* 1}$ Nurulazlina Ramli, Anis Fariza Md. Pazil, Noor Farhana Halil Abdul Razak

${ }^{1}$ Centre of Advanced Electrical and Electronic Systems (CAEES),

Faculty of Engineering, Built Environment \& IT, SEGi University

* azlinaramli@segi.edu.my

\begin{abstract}
This paper presents a Reconfigurable Stacked Patch Microstrip Antenna (RSPMA) uses a combination of aperture coupled technique and stacked patch technology for reducing feigned radiation patterns. This RSPMA consists of three FR-4 substrate layers with $4.7 \mathrm{~mm}$ thickness each and $3 \mathrm{~mm}$ thick of an air-filled substrate between stacked patches and the ground plane. The top patches are rectangular shaped with a T-shaped hole at the center and the bottom patches are T-shaped, both etched on top of substrate 1 and substrate 2 respectively act as a radiating element. Two H-shape designs of aperture slots of different sizes on the ground plane are positioned at the center of the ground plane with reference to the top patches and bottom patches. A copper strip is used as an ideal RF switch is implemented at the feed line network for the ON and OFF mode. Thus, by adjusting the switch mode, the resonance frequencies can be varied, thus frequency reconfigurable is achieved. The activation of the selected aperture slots will produce waves and radiates the signal to the radiating layers of the patch antenna. Hence, two different frequencies, either at $1.8 \mathrm{GHz}$ or $2.3 \mathrm{GHz}$ were achieved through the RSPMA with the gain of $3.691 \mathrm{~dB}$ and $2.291 \mathrm{~dB}$, respectively. The antenna designed was simulated using CST Microwave Studio at an operating frequency of $1 \mathrm{GHz}$ to $2.7 \mathrm{GHz}$. The RSPMA is said to be potentially beneficial to the wireless communication system.
\end{abstract}

Keywords: Aperture Coupled, Aperture Slots, Reconfigurable, RF Switch, Stacked 


\section{Introduction}

Nowadays, reconfigurable microstrip antennas (RMA) have received great attention on the modern wireless communication system (K. Chung et al, 2020). High demands on the use of antennas which is capable of accessing services in various frequency bands have led the researcher to implement reconfigurable antenna in their design. This is because they can offer the diversity of performances related to the operating frequency, polarization, and radiation pattern to improve the communication quality and capacity (Ying Liu et al, 2020). Reconfigurable microstrip antenna has disclosed several advantages and capabilities in the world compared to a conventional antenna where it allows supporting multiple operating frequencies (M. T. Ali et al, 2020). Moreover, a reconfigurable antenna will be an attractive feature in the modern wireless communication systems because it can achieve a multifunction antenna by using a single antenna (S. Muhamud @ Kayat et al, 2012).

By configuring the switching components such as PIN diodes (A. I-Yasir et al, 2019), varactor diodes (T. Yamagajo et al, 2019), MEMs switches, and optoelectronic switches, the antenna can be operated at different operating frequencies. By configuring the RF switches either to ON or OFF mode, the antenna can achieve reconfigurability. According to (D. Ramakrishna et al, 2011) the concept of a rotatable patch that contains different sizes and shapes of the patch in the same radiating layer led to the frequency reconfigurability. Every rotation at a different antenna structure is fed to produce a different frequency range. Besides, various shapes of slots have been embedded on patch antennas to reduce their size. Another technique to achieve frequency reconfigurable is by modifying the position of the connecting element on the antenna patches (Kordzadeh, A. and F. Hojat Kashani, 2009). Recently, a microstrip antenna with Koch shaped fractal defects was presented in which $85 \%$ size reduction was achieved (K. Surmeli and B. Turetken, 2011). As based on (S. K. Sharma and M. Rattan, 2010) microstrip antennas' drawback is narrow bandwidth. Therefore, to overcome this drawback, several methods have been applied to achieve frequency reconfigurable by multilayer stacked patch technique (N. Ramli et al, 2013). These include using parasitic elements 
on the same layer or another layer called stacked, using various slot shapes such as U-slot, using a bending probe, and using shorting pin or shorting wall structures. However, in (N. Ramli et al, 2012) the author invented a new coupling method by using the combination of an aperture coupled technique and stacked patch technology to achieve frequency reconfigurable. The advantages of this technique are able to increase the gain performances and reduced spurious radiation patterns. Apart from that, its manufacturing cost is very economical in which size is smaller, compact, and easy to fabricate.

The focus of this paper is to apply and prove the concept of designing a reconfigurable antenna by using the combination of an aperture coupled technique and stacked patch technology using different shapes of the antenna. Next, to design the RSPMA integrated with ideal RF switch to achieve different operating frequencies at $1.8 \mathrm{GHz}(\mathrm{ON}$ mode) and $2.3 \mathrm{GHz}(\mathrm{OFF}$ mode). An ideal switch which is a copper strip is used based on the basic concept of the RF switch configuration. The current flowing from the length of the feedline network will activate particular aperture slots on the ground plane and radiates the waves to selected patches at different substrate layers. Therefore, the feigned radiation from the feedline and radiating patch can be reduced and eliminated. In section 2, the procedure and methods of RSPMA, antenna design and frequency reconfiguration are explained. In section 3 , the antenna performances in terms of the frequency, return loss, gain and the characteristic of the radiation pattern was compared between simulations and measured results. Finally, the conclusions are presented in section 4 .

\section{Methodology}

Two antenna structures, with and without ideal PIN diode switch which is a copper strip has been used are then fabricated to be evaluated and compared with the simulated results. The antenna's performance in terms of the frequency, return loss, gain and the characteristics of the radiation pattern were obtained and analyzed by using the CST simulation. The procedures and methods to be used to achieve the project objectives are shown in Fig. 1 as follows. 


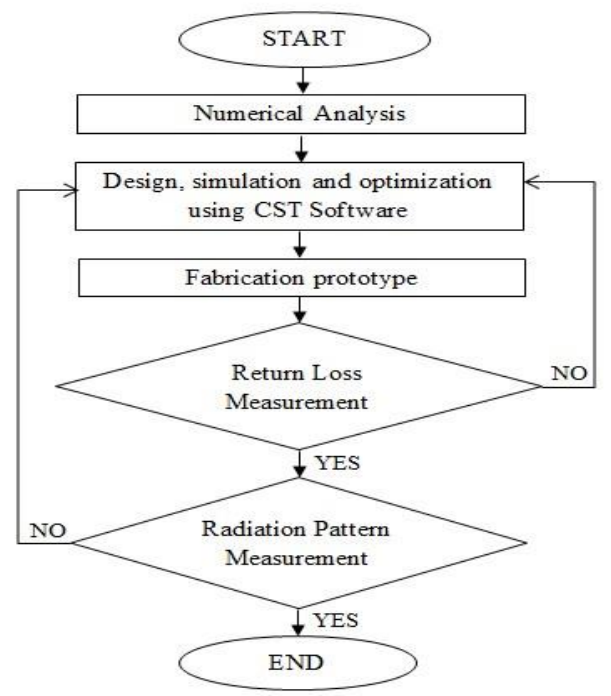

Figure 1. Flowchart of the RSPMA design

Next, the antenna design is fabricated based on the simulation antenna structure and dimension. Then, the prototype RSPMA is validated by measuring the return loss, Voltage Standing Wave Ratio (VSWR), and radiation pattern using the Vector Network Analyzer (VNA) and anechoic chamber.

The configuration of the RSPMA structures consists of three substrate layers as shown in Fig. 2. This RSPMA uses a combination of aperture coupler technique and stacked technology, where feedline and the radiating elements which are patches are located at different substrate layers separated by an air gap. The thickness of the air gap substrate is $\mathrm{h}=3 \mathrm{~mm}$ with a dielectric constant of $\varepsilon r=1$ and tangent loss, $\delta=0.0001$. The radiating elements, known as the top patch and bottom patch are printed on top of substrate 1 and substrate 2 respectively. Both patches and feedline are printed on FR-4 substrates which has a dielectric constant, $\varepsilon r=4.7$, the thickness of $1.6 \mathrm{~mm}$ and tangent loss, $\delta$ $=0.025$. While the ground plane is located between the substrate 3 (feedline substrate) and an air-filled gap. At the back of the feedline substrate, a feed network is integrated with an RF switch. The entire substrate dimension is $58.96 \mathrm{~mm} \times 47.77 \mathrm{~mm}$ and the switch size at the feedline is $0.9 \mathrm{~mm} \times 3.979$ $\mathrm{mm}$. At the middle layer in-between substrate 3 and air gap, two different sizes 
of aperture slots H-shape were designed at the ground plane. The aperture slot must be small and located at the center of the patches to produce a maximum coupling. Typically, the ratio of slot length to width is $1 / 10$. In addition, a 50ohm feedline characteristic impedance was fed to this antenna.



(a)

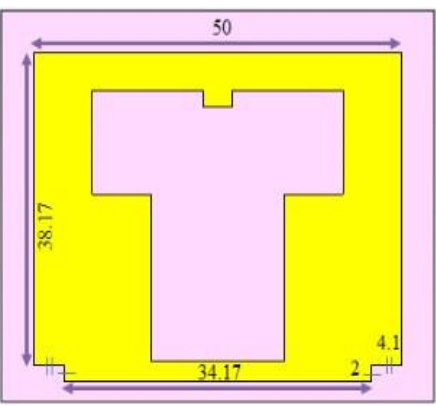

(b)

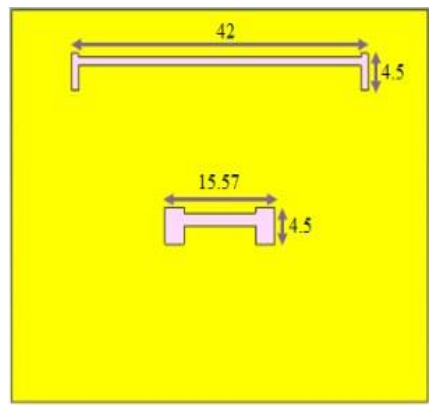

(d)

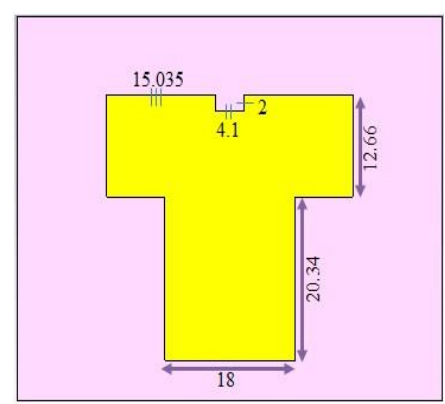

(c)

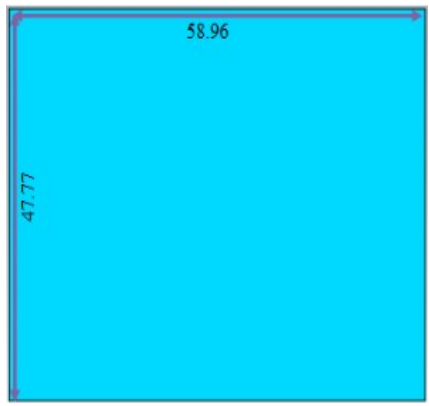

(e) 




(f)

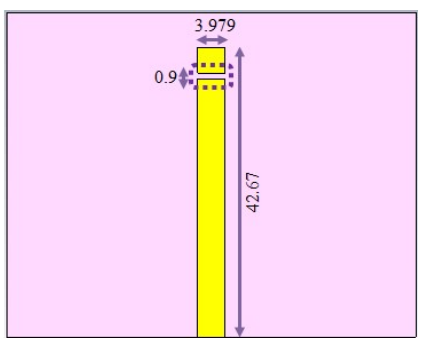

(g)

Figure 2. Overall structure of RSPMA (a) side view, (b) top patch view, (c) bottom patch view, (d) ground patch view, (e) air gap, (f) feedline back view (ON mode), and ( $\mathrm{g}$ ) feedline back view (OFF mode)

Basically, bottom and top patch are designed with a basic rectangular shape where the bottom patch operates at $2.3 \mathrm{GHz}$ while the top patch is operating at $1.8 \mathrm{GHz}$. There are several equations that need to be considered to configure the length and width of both patches (Balanis, 1997). Table 1 shows the dimensions of the basic rectangular of RSPMA between theoretical and optimization. In addition to that, some parts of the patches are modified to meet the needs of antenna requirements.

Table 1. Dimension of RSPMA structure

\begin{tabular}{|l|c|c|c|c|}
\hline & \multicolumn{2}{|c|}{ Top Patch: } & \multicolumn{2}{c|}{$\begin{array}{c}\text { Bottom Patch: } \\
\text { Frequency at 1.8 GHz }\end{array}$} \\
\cline { 2 - 5 } & $\begin{array}{c}\text { Theoretical } \\
(\mathrm{mm})\end{array}$ & $\begin{array}{c}\text { Optimized } \\
(\mathrm{mm})\end{array}$ & $\begin{array}{c}\text { Theoretical } \\
(\mathrm{mm})\end{array}$ & $\begin{array}{c}\text { Optimized } \\
(\mathrm{mm})\end{array}$ \\
\hline Width, W & 49.36 & 50.00 & 38.63 & 34.17 \\
\hline Length, L & 38.17 & 40.17 & 29.76 & 33.00 \\
\hline $\begin{array}{l}\text { Width of the feed line, } \\
\text { Wf }\end{array}$ & 2.93 & 3.979 & 2.93 & 3.979 \\
\hline Length of feedline, Lf & 38.12 & 41.77 & 42.00 & 42.67 \\
\hline Width of the ground, Wg & 58.96 & 58.96 & 48.23 & 58.96 \\
\hline Length of ground, Lg & 47.77 & 47.77 & 39.36 & 47.77 \\
\hline
\end{tabular}

An ideal RF switch which is a copper strip is used to control the electrical flow from the feedline to activate the respected aperture slots and patches. During the ON-mode, the coupled energy from both aperture slots will activate both patches. As referred to Fig. 3(a), the top patch and bottom patch were combined and connected each other and become the large patch resulting in a 
lower resonant frequency of $1.8 \mathrm{GHz}$. In contrast, during the OFF-mode Fig. 3(b), only a small aperture slot is activated and coupled with the energy to bottom patch. In this mode, the activation of the bottom patch resulting in the antenna operating at a high resonant frequency of $2.3 \mathrm{GHz}$.

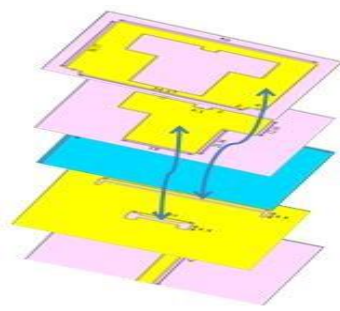

(a)

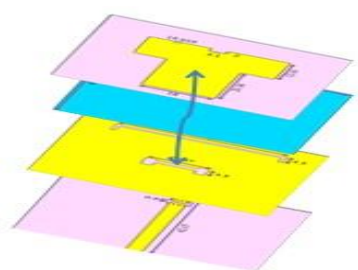

(b)

Figure 3. Technical operation of the RSPMA during (a) ON mode and (b)

OFF mode

\section{Simulation and experimental results}

The Computer Simulation Technology (CST) Microwave Studio is used to simulate the RSPMA. Fig. 4 shows the simulated surface current in the feed network in different switching modes. When the RF switch is ON, both aperture slots are activated, hence the length of the feed network at the back of the antenna is fully connected. Fig. 4(a) shows the current travels from the SMA connector to both aperture slots. Each aperture slot will produce the waves to activate the top and bottom patch respectively. During this time, the antenna will operate at $1.8 \mathrm{GHz}$. As opposed to when RF switch is OFF, only a small aperture slot is activated, and the waves will radiate to activate the bottom patch. During this mode, the antenna has the capability to operate at $2.3 \mathrm{GHz}$ as shown in Fig. 4(b). The simulated and measured graphs of the return loss during $\mathrm{ON}$ and $\mathrm{OFF}$ mode are shown in Fig. 5. During the ON mode, the antenna operated at $1.8 \mathrm{GHz}$ with a return loss of $19.39 \mathrm{~dB}$ achieved by simulation while $-26.82 \mathrm{~dB}$ is achieved by measured using a VNA analyzer. Meanwhile, during the OFF mode, the antenna operated at frequency $2.3 \mathrm{GHz}$ with a return loss of $-17.67 \mathrm{~dB}$ achieved by simulation, and $-15.12 \mathrm{~dB}$ is achieved during the measurement process. From Fig. 5(a) and (b), there is a good agreement between the simulated and measured results for both modes. 


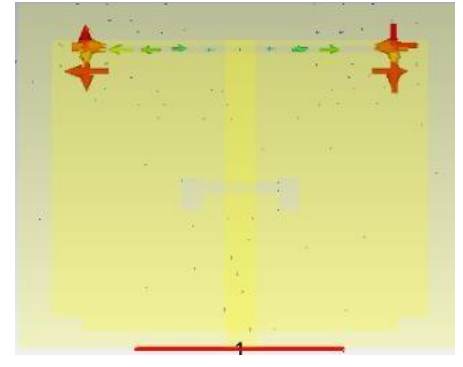

(a)

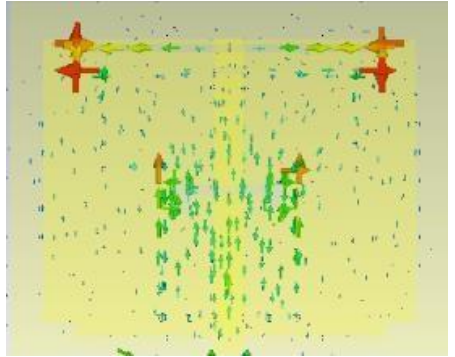

(b)

Figure 4. Simulated results of the surface current from feed network of the RSPMA during (a) ON mode at $1.8 \mathrm{GHz}$ and (b) OFF mode at $2.3 \mathrm{GHz}$

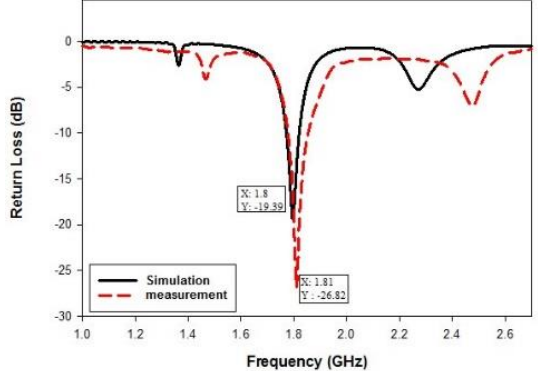

(a)

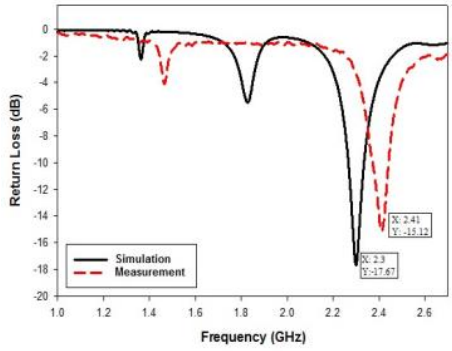

(b)

Figure 5. Simulated and measured return losses during (a) ON mode and (b)

OFF mode

Table 2 shows the frequencies, return losses, and a VSWR result between simulated and measured results during the ON and OFF modes of the switch. It is clearly shown that during ON mode the measured results are well operated at the desired frequency as it is approximately similar to the simulated result which is $1.81 \mathrm{GHz}$. However, the measured result during the OFF mode is slightly shifted about $110 \mathrm{MHz}$ from the desired frequency of $2.3 \mathrm{GHz}$ with VSWR of 1.42. In terms of return loss value, the measured result for ON mode is much better as compared to simulate a result which is $-26.82 \mathrm{~dB}$. While, for OFF mode, the return loss for measuring results is a bit lower compared to the simulated results which is $-17.67 \mathrm{~dB}$. This is due to the effects of the waves from the activation of the selected aperture slots during ON and OFF modes. During the ON mode, both aperture slots are activated, and the waves will radiate directionally towards both patches, resulting in the measured frequency 
to be approximately the same as the simulated frequency and have good return loss value. While during the OFF mode, only one aperture slot is activated, and the waves will radiate to activate the bottom patch. Thus, the bottom aperture slot is not able to couple the energy maximized and led to the frequency to be shifted to $2.41 \mathrm{GHz}$ and return loss with $-15.12 \mathrm{~dB}$.

Table 2. Comparison of simulated and measured results between on and off

\begin{tabular}{|c|c|c|c|c|}
\hline \multirow{2}{*}{ Parameter } & \multicolumn{2}{|c|}{ ON mode } & \multicolumn{2}{c|}{ OFF mode } \\
\cline { 2 - 5 } & Simulated & Measured & Simulated & Measured \\
\hline Resonant frequency $(\mathrm{GHz})$ & 1.8 & 1.81 & 2.3 & 2.41 \\
\hline S11 (dB) & -19.39 & -26.82 & -17.67 & -15.12 \\
\hline VSWR & 1.24 & 1.09 & 1.3 & 1.42 \\
\hline
\end{tabular}

modes

Table 3 shows the characteristics of the radiation pattern between $\mathrm{ON}$ and OFF mode switch during the simulation. The HPBW at $1.8 \mathrm{GHz}$ is $98.72^{\circ}$ and at $2.3 \mathrm{GHz}$ is $91.2^{\circ}$. Fig. 6 shows the simulated and measured radiation pattern for different RF switch configuration. As referred to Table 3, the main lobe magnitude for $\mathrm{ON}$ mode is $3.69 \mathrm{~dB}$ which is higher than during OFF mode which is $1.84 \mathrm{~dB}$. This is clearly shown in Fig. 6(a) and Fig. 6(b). It shows that the signal strength during ON mode is stronger than during OFF mode. Thus, the measured radiation pattern obtained is approximately similar to the simulation radiation pattern result. As referred to Fig. 6(b), the side lobe of radiation pattern of OFF mode during measurement (shown in red medium dash colour line) is slightly bigger than the simulation radiation pattern. This is due to the activation of single aperture slots at the center of the ground plane which is unable to have maximum energy coupling. In spite of that, the main beam direction is said to be directed to $0^{\circ}$ for both modes. In addition of that, the $3 \mathrm{D}$ radiation pattern shows the signal strength is mode is $2.291 \mathrm{~dB}$ respectively. By the implementation of the stacked patch technology, directional towards the radiating patches as shown in Fig. 7(a) and in Fig. 7(b). The gain for ON mode is $3.691 \mathrm{~dB}$ while OFF the gain achieved by the RSPMA is considerably high. Fig. 8 shows the prototype of RSPMA which have been measured in an anechoic chamber and by using VNA analyzer. 
Table 3. Simulated radiation pattern characteristics during on and off modes switch

\begin{tabular}{|l|c|c|}
\hline \multirow{2}{*}{\multicolumn{2}{|c|}{ Characteristics }} & \multicolumn{2}{|c|}{ Switch mode } \\
\cline { 2 - 3 } & ON & OFF \\
\hline Resonant Frequency $(\mathrm{GHz})(\mathrm{GHz})$ & 1.8 & 2.3 \\
\hline Return Loss $(\mathrm{dB})$ & -19.39 & -17.67 \\
\hline Bandwidth $(\mathrm{MHz})$ & 51.3 & 68.1 \\
\hline Main lobe level $(\mathrm{dB})$ & 3.69 & 1.84 \\
\hline Side lobe level $(\mathrm{dB})$ & -2.9 & -3.1 \\
\hline Gain $(\mathrm{dB})$ & 3.691 & 2.291 \\
\hline HPBW $\left(^{\circ}\right)$ & 98.72 & 91.20 \\
\hline
\end{tabular}

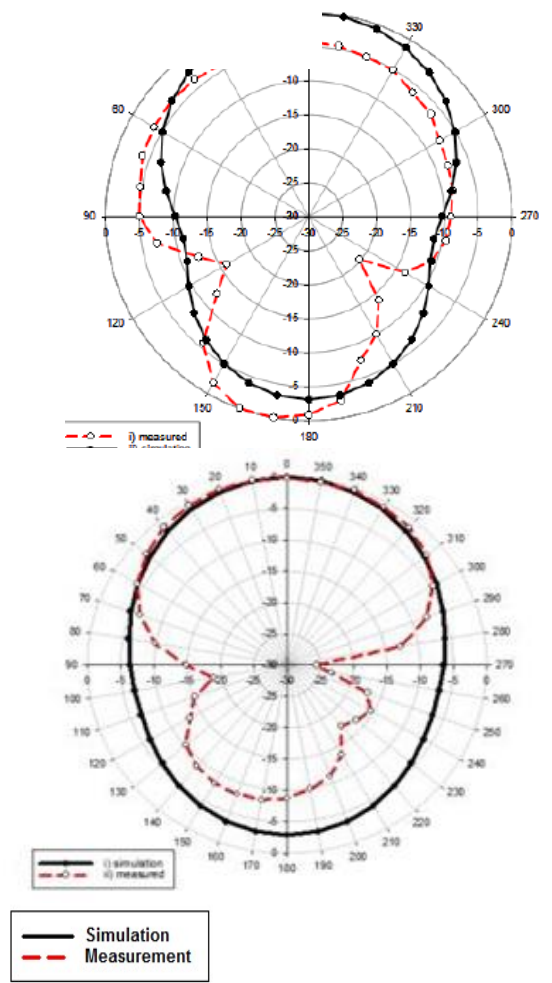

(a) (b)

Figure 6. Radiation pattern of the RSPMA during simulation and measured (a) ON mode at $1.8 \mathrm{GHz}$ and (b) OFF mode at $2.3 \mathrm{GHz}$ 




(a)

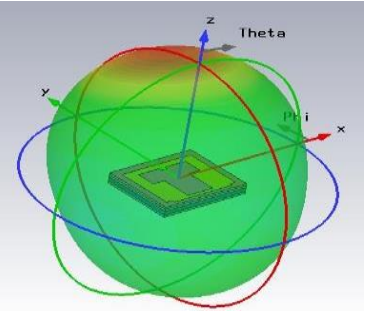

(b)

Figure 7. Simulated radiation pattern in $3 \mathrm{D}$ plot during (a) $\mathrm{ON}$ mode at 1.8

$\mathrm{GHz}$ and

(b) OFF mode at $2.3 \mathrm{GHz}$

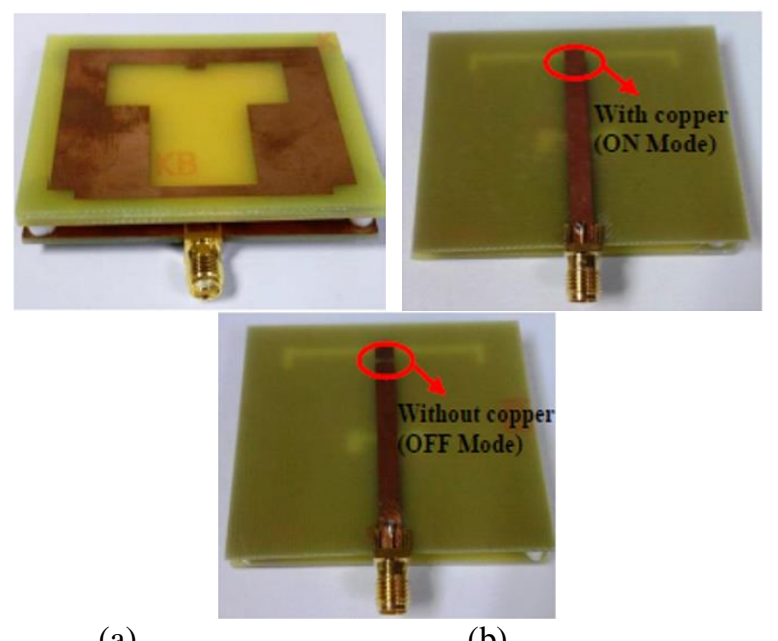

(a)

(b)

(c)

Figure 8. Prototype of the RSPMA (a) top view, (b) back view during switch is $\mathrm{ON}$ mode, and (c) back view during switch is OFF mode

\section{Conclusion}

In this paper, a reconfigurable stacked patch microstrip antenna was designed. The concept of designing RSPMA using the combination of an aperture coupled technique and stacked patch technology was applied and prove that it can be implemented by using different antenna shapes to achieve the frequency reconfigurable. A reconfigurable stacked patch microstrip antenna integrated with ideal RF switch which frequency operated at $1.8 \mathrm{GHz}$ and $2.3 \mathrm{GHz}$ was designed. On top of that, the RSPMA has a unique structure where the radiating patches are sorted in a multilayer structure called stack and 
consists of two different sizes of H-shapes of the aperture slots placed on the ground plane. The simulated and measured results using the copper strip as ideal RF switch at the feed network is reconfigured for ON and OFF mode to activate the selected aperture slots on the ground and the respected radiating patch. The activation of the selected aperture slots will affect the excitation of the resonant frequency either at $1.8 \mathrm{GHz}$ or $2.3 \mathrm{GHz}$. Hence, it proves that RSPMA can do frequency reconfigurability. Instead of that, by using the RSPMA, it is achieving a multifunction antenna by using a single antenna.

\section{Acknowledgement}

The authors would like to thank the Faculty of Engineering, Built Environment \& Information Technology (FoEBEIT), SEGi University, Kota Damansara for the financial support and the Antenna Research Centre (ARC), Faculty of Electrical Engineering, Universiti Teknologi MARA (UiTM), Shah Alam, Malaysia for the measurement support.

\section{References}

A. I-Yasir, Naser Ojaroudi Parchin, Ali Alabdullah, Widad Mshwat, Atta Ullah and Raed Abd-Alhameed(2019), "New Pattern Reconfigurable Circular Disk Antenna Using Two PIN Diodes for WiMax/WiFi (IEEE 802.11a) Applications," 2019 16th International Conference on Synthesis, Modeling, Analysis and Simulation Methods and Applications to Circuit Design (SMACD).

Balanies, C. A., Antenna Theory: Analysis \& Design, 2nd edition, John Wiley \& Sons, Inc. 1997.

D. Ramakrishna, M. Muthukumar, and V.M. Pandharipande(2011), "Design and realization of Rectangular Reconfigurable Antenna (RRA) for airborne RADAR," IEEE InternationalConference on RF and Microwave (RFM), pp.127-132.

Kordzadeh, A. and F. Hojat Kashani(2009), “A new reduced size microstrip patch antenna with fractal shaped defects," Progress In Electromagnetics Research B. 11,pp 29-37.

K. Chung, Y. Nam, T. Yun, and J. Choi(2020), "Ultralow-Profile, Electrically Small, Pattern-Reconfigurable Metamaterial-Inspired Huygens Dipole Antenna", IEEE Transactions on Antennas and Propagation,3(68) pp. $1238-1248$.

K. Surmeli and B. Turetken(2011), "U-slot stacked patch antenna using high and low dielectric material combinations in S-band," URSI Gen. Assem. Sci. Symp., pp. 1-4. 
M. T. Ali, et al.(2020), "Differential Frequency-Reconfigurable Antenna Based on Dipoles for Sub-6 GHz 5G and WLAN Applications", IEEE Antennas and Wireless Propagation Letters,3(19).

N. Ramli, M. T. Ali, A. L. Yusof, S. Muhamud-Kayat, A. A. A. Aziz(2013), "PIN diode switches for frequency-reconfigurable stacked patch microstrip array antenna using aperture-coupled technique," Asia-Pacific Microwave Conference Proceedings (APMC).

Nurulazlina Ramli, Mohd Tarmizi Ali, Azita Laily Yusof, Suzilawati Muhamud-Kayat, Hafiza Alias(2013), "Aperture-coupled frequencyreconfigurable stacked patch microstrip antenna (FRSPMA) integrated with PIN diode switch," Progress In Electromagnetics Research C. 39, pp. 237-254.

S. K. Sharma, M. Rattan(2010), “'Analysis of Broadbanding and Minimization Techniques for Square Patch Antenna', IETE Journal of Research, 2(56).

S. Muhamud @ Kayat, M. T. Ali, M. K. M. Salleh (2012), “Truncated rhombic microstrip patch array antenna with frequency reconfigurability, " IEEE Asia Pacific Conference on Applied Electromagnetics (APACE), 11-13.

T. Yamagajo, and Y. Koga (2019), “A Frequency-Tunable Dual-Band SingleLayer Shorted Multi-Ring Microstrip Antenna Fed by an L-probe with Varactor Diodes," 2019 IEEE International Symposium on Antennas and Propagation and USNC-URSI Radio Science Meeting.

Ying Liu, Qia Wang, Yongtao Jia, and Peisheng Zhu (2015), “A Frequencyand Polarization- Reconfigurable Slot Antenna Using Liquid Metal," IEEE Transactions on Antennas and Propagation. 